Gut, 1972, 13, 559-565

\title{
Effect of luminal ions on the transepithelial electrical potential difference of human rectum
}

\author{
E. Q. ARCHAMPONG AND C. J. EDMONDS \\ From the Departments of Surgery and of Clinical Research (MRC), University College Hospital Medical \\ School, London
}

SUMMARY Skin electrodes are the most convenient reference electrodes for clinical measurements of electrical potential differences (pd) across the epithelium of the alimentary tract but the presence of an electrical charge on normal skin introduces an error. In the present study, by comparison with results obtained using subcutaneous and intravenous electrodes, it was shown that an intradermal injection of saline abolished the skin potential differences. This simple method, therefore, allows skin electrodes to be used to measure the true transepithelial potential differences of gut mucosa. The method was applied to investigate the effect on the rectal potential difference of altering the composition of the luminal solutions. Changes in the cations (sodium, potassium, magnesium) showed that sodium was the most important cationic determinant of the potential difference, especially when sodium absorption was stimulated by giving mineralocorticoids. Changes in the anions (chloride, iodide, bromide, nitrate, bicarbonate, sulphate, phosphate, citrate, and acetate) indicated that the molecular size of the anion rather than its chemical nature was the significant factor and suggested that the ions had to cross a barrier relatively impermeable to anions of radius greater than 3.5 to $4 \mathrm{~A}^{\circ}$. Changes in osmolality and glucose concentration were without effect.

Rectal and colonic epithelium are electrically polarized, the luminal side being generally negatively charged with respect to the serosal side. A simple technique was recently described which could be used in man on a routine basis to measure the electrical potential difference of rectal and colonic epithelium. The original method was adequate for showing the rise induced by aldosterone (Edmonds and Godfrey, 1970) and the fall of potential difference in colitis (Edmonds, 1970) but it tended to underestimate to some extent the true transmucosal potential difference. This was a consequence of using the perianal skin surface as the reference site which, although convenient for clinical investigations, involves an error due to the electrical charge on the skin. A potential difference is present across normal skin, the surface being negatively charged with respect to the subcutaneous tissue, and consequently the original method gave a value for the transmucosal potential difference which underestimated its true value by an amount equal to the perianal potential difference since the latter is effectively in series with that across the epithelium. The perianal

Received for publication 21 March 1972. skin potential difference was usually found to be relatively low (about 10 to $15 \mathrm{mV}$ ) in comparison with most other regions and considerably less than that across the colonic epithelium. High values (over $20 \mathrm{mV}$ ) were, however, sometimes observed.

It would clearly be preferable to eliminate the skin potential difference and obtain the true transmucosal potential difference. Unfortunately, to achieve this consistently and reliably, the reference electrode has had to be placed intravenously or subcutaneously (Anderssen and Grossman, 1965; Geall, Spencer, and Phillips, 1969; Grantham, Code, and Schlegel, 1970). This involves the use of sterile electrodes and an appreciably more complicated procedure than is suitable for routine clinical work. A variety of methods have been employed in an attempt to reduce the skin charge to allow measurement of gastrointestinal potential differences by skin electrodes. These procedures do not usually do more than reduce the skin charge, and for consistent results considerable damage to the skin by abrasive or skin drill procedures is needed since most of the skin electrical resistance is in the germinating layer (Edelberg, 1968).

The purpose of the present report is twofold. 
First, to describe a simple, atraumatic method of eliminating skin charge and so allow the use of skin electrodes to measure the true transmucosal potential difference. Secondly, to apply the method to examine the effect on it of varying the composition of the luminal solution.

\section{Methods}

SUBJECTS

The patients investigated were drawn principally from a group undergoing investigation of the alimentary tract. Results are reported only from patients with no evidence of organic bowel disease and informed consent for the procedure was obtained in all cases.

\section{ELECTRODES}

The electrodes were constructed of perspex (lucite) and contained silver-silver chloride junctions prepared as described by Donaldson (1958). The design of electrodes is shown in Figure 1. For intravenous or subcutaneous use, the electrode (A) which had a Luer fitting, was plugged into a nylon cannula (Plextrocan, Portex Ltd) which contained normal saline and had been placed into the antecubital vein or inserted just beneath the skin. A similar design

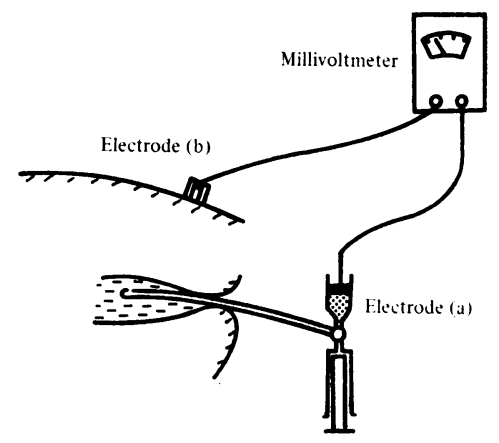

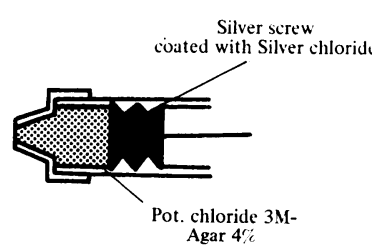

(a)

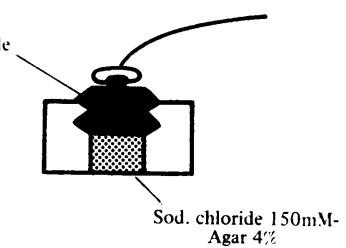

(b)
Fig. 1 Diagrammatic representation of the method. a Electrode connected to intravenous or subcutaneous cannulae when used as a reference electrode. A similar electrode was connected to a catheter in the rectal lumen for use as the luminal side electrode. b Skin reference electrode. of electrode was used for connecting to the solution in the gut lumen. It was filled with $3 \mathrm{M}$ potassium chloride/agar $4 \%$ in these experiments in order to minimize the changes in junction potentials which could occur when the luminal solution was altered in its composition. The skin electrodes usually contained $150 \mathrm{Mm}$ sodium chloride/agar $4 \%$ which is as satisfactory in this situation as $3 \mathrm{M}$ potassium chloride and such electrodes are considerably easier to preserve. The asymmetry between the electrodes filled with $150 \mathrm{mM}$ sodium chloride and $3 \mathrm{M}$ potassium chloride was considerable (approximately $60 \mathrm{mV}$ at room temperature) but constant. At the beginning and end of each experiment the two electrodes used were placed together in a beaker containing a little $150 \mathrm{mM}$ sodium chloride solution at room temperature to determine the asymmetrical potential difference and observations were corrected for this asymmetry. The electrodes containing the silver-silver chloride junctions were at room temperature during the experiments as they were not inserted into the rectum. Some temperature gradient must, however, have been present in the solutions as those in the rectum were in continuity with those in the cannula. Preliminary model experiments showed, however, that the error in measurement of the potential difference introduced by the temperature gradient was small, at maximum about 1 to $2 \mathrm{mV}$. Consequently no attempt has been made to correct for this. In stating the observed values of transmucosal potential difference, the polarity given always refers to that of the luminal side of the mucosa.

\section{METER}

Leads from the electrodes were connected to a portable battery operated millivoltmeter. ${ }^{1}$

\section{SKIN PREPARATION}

The skin was cleaned with surgical spirit and about $\mathbf{0} \cdot 1-0.2 \mathrm{ml}$ of sterile isotonic sodium chloride solution injected intradermally through a fine needle to produce a bleb about $1 \mathrm{~cm}$ diameter. A little lignocaine 1/5000 was added to the saline to eliminate the discomfort of the injection. The skin electrode was applied so that the exposed agar-saline column rested on the bleb. The electrode was held in position by a strip of adhesive plaster (Fig. 1). In most experiments, the skin electrode was applied to the outer part of the right thigh as this was conveniently exposed with the patient in the left lateral position. In some experiments the skin electrode was placed on the forearm; there was no difference between these sites as regards the mucosal potential differences measured.

${ }^{1}$ Manufactured by Mauritex Ltd, Macclesfield, Cheshire. 
PROCEDURE

With the subject lying on his left side, a PVC cannula (diameter $4 \mathrm{~mm}$ ) was introduced into the rectum so that its tip was about 8 to $10 \mathrm{~cm}$ from the anus. Twenty $\mathrm{ml}$ of solution was injected into the rectum, the syringe removed and the electrode plugged into the end of the cannula-the fluidfilled cannula thus made connexion with the solution within the bowel lumen (Fig. 1). When the potential difference was stable, it was recorded and the electrode then removed and the solution drained out of the rectum through the cannula. The next solution was introduced and the procedure repeated. In the initial experiments to determine the adequacy of the intradermal saline bleb method in eliminating the skin potential difference, the potential difference was measured between the intraluminal solution and reference electrodes placed in various positions, intravenously, subcutaneously, on skin cleaned with spirit alone and on skin injected with saline.

\section{SOLUTIONS}

All solutions were prepared freshly before each experiment from a series of stock solutions made from Analar reagents.

DRUGS

Aldosterone, $\mathbf{0} \cdot 25 \mathrm{mg}$ (Aldocorten, Ciba), was given in two im injections at a two-hourly interval, the first injection being eight hours before measurements. Fludrocortisone (9- $\alpha$-fluorocortisol), $0.5 \mathrm{mg}$, was given in two oral doses at 12 and 24 hours before measurements.

\section{Results}

EFFECT OF INTRADERMAL SALINE INJECTION In initial experiments on six individuals, the potential difference on the skin itself was measured before and after intradermal injection of saline. A salinefilled cannula was inserted subcutaneously in the outer aspect of the thigh and connected to an electrode of the type shown in Figure 1a. The skin electrode was then placed on the skin surface and the skin potential difference measured. It averaged $-22 \pm 3$ (SD) $\mathrm{mV}$. The skin electrode was removed, saline was injected, and the skin electrode reapplied over the bleb. On remeasuring, the skin potential difference averaged $-1 \mathrm{mV}$ (range +1 to $-3 \mathrm{mV}$ ).

In a subsequent study on 12 individuals, the rectal potential difference as determined by our original method (using perianal skin as the reference site) was compared with that measured using an intravenous electrode or an electrode placed on injected skin. By the original perianal skin reference method, the average rectal potential difference for these subjects was $25 \pm 7 \mathrm{mV}$, agreeing well with previous results (Edmonds and Godfrey, 1970). Using the intravenous reference electrode, the potential difference was $41 \pm 7 \mathrm{mV}$ and with a skin electrode on injected skin was $40 \pm 7 \mathrm{mV}$. In nine of the subjects, the difference between the intravenous and skin methods did not exceed $1 \mathrm{mV}$, in two subjects it was $2 \mathrm{mV}$, and in one $3 \mathrm{mV}$. Intradermal saline injection appears therefore to be an effective method of virtually abolishing the skin potential difference. It is important that the injection is made intradermally; saline injected subcutaneously was found to be ineffective. The effect was achieved within a few seconds of injecting the saline, lasted for at least 10 minutes and often considerably longer.

\section{EFFECT OF VARYING THE COMPOSITION OF \\ THE LUMINAL SOLUTION \\ Cations}

When the sodium chloride concentration of the luminal solution was reduced from 100 to 10 m-equiv/l, the potential difference usually fell (Fig. 2) but the change was relatively small (4 to $11 \mathrm{mV})$. In those individuals previously given aldosterone or fludrocortisone, the potential differences observed were greater than in the other subjects at each sodium concentration used and the change in potential difference was greater ( 15 to $28 \mathrm{mV})$. When sulphate, a larger, relatively impermeable anion, was substituted for chloride, these effects were more obvious, the potential difference changing by 7 to $17 \mathrm{mV}$ in those not given a mineralocorticoid and by 26 to $34 \mathrm{mV}$ in those given a mineralocorticoid (Fig. 2). As there appeared to be no difference whether aldosterone or fludrocortisone was used, these have not been considered separately. In some experiments, solutions containing only $1 \mathrm{~m}$-equiv/1 of sodium were used (Fig. 3). Even with so little sodium present, the mucosa was still charged with the lumen negative but now there was little difference whether sulphate or chloride was the anion present or whether the subject had been treated with mineralocorticoids or not.

In experiments in which the potassium and magnesium concentrations were changed, sulphate was used as the anion and the sodium concentration, at $20 \mathrm{~m}$-equiv/l, was constant in all solutions. Increasing the potassium concentration sometimes produced a small rise in potential difference ( 3 to $8 \mathrm{mV}$ in three individuals, but no definite change in two). Increasing the magnesium concentration produced only small and inconsistent changes and on average had no significant effect (Fig. 2). Previous administration of fludrocortisone did not influence the responses to potassium or magnesium. 


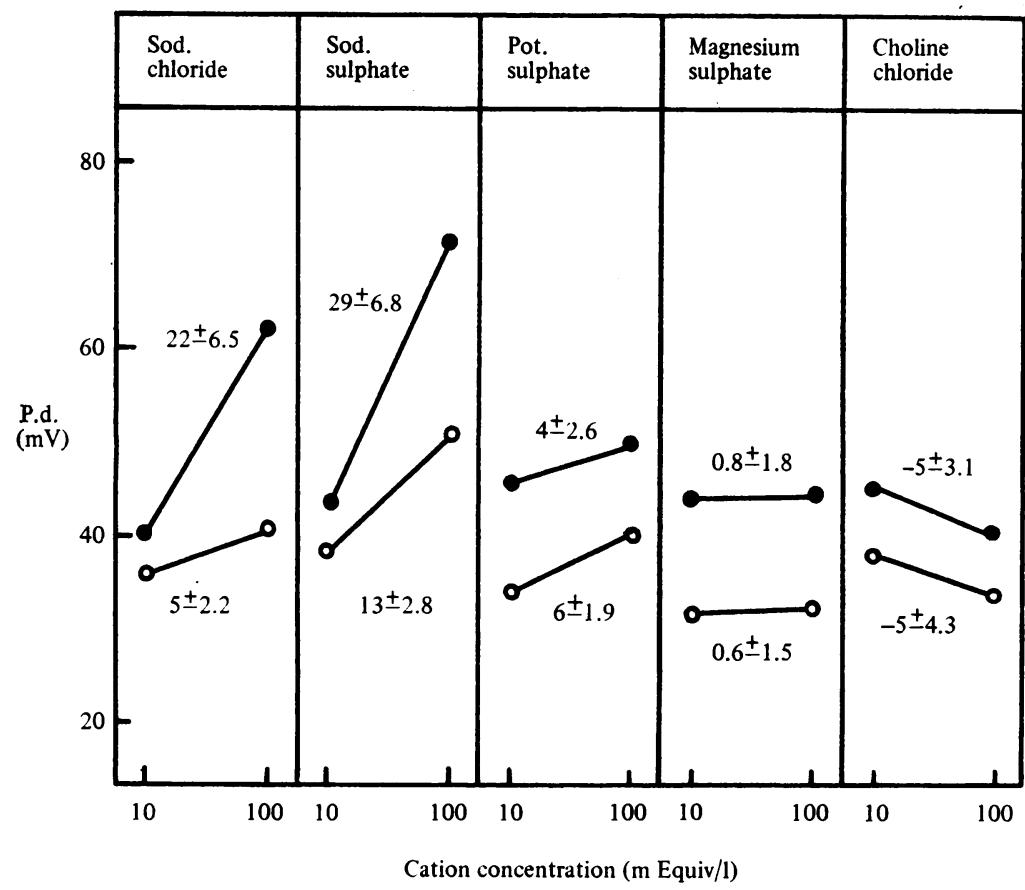

Fig. 2 Changes in potential difference produced by altering luminal ionic composition. All solutions were rendered isosmotic by adding mannitol, and with magnesium sulphate and choline chloride the solutions also contained sodium sulphate 20 m-equiv/l. $\bigcirc$, six normal individuals; -, four individuals previously given aldosterone or fludrocortisone.

Mean values are shown together with the mean change $\pm 1 S D$.

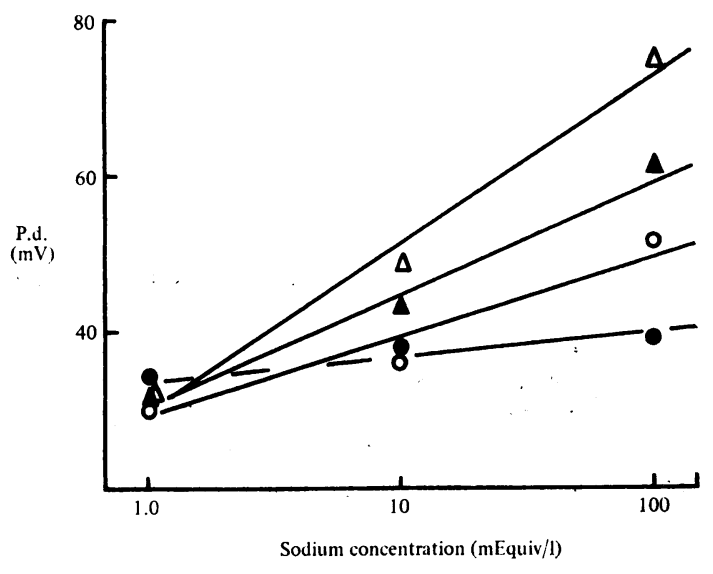

Fig. 3 Changes in potential difference in a normal subject in relation to luminal sodium concentration examined before $(Q, 0)$ and after fludrocortisone $(\triangle, \Delta)$ administration. Choline chloride was substituted for sodium, chloride and mannitol for sodium sulphate when the sodium concentration was reduced. (Sodium chloride is represented by solid symbols and sodium sulphate by open symbols.)

\section{Anions}

The effect of altering the chloride concentration in the lumen was examined using choline chloride, the solutions all containing sodium sulphate at a concentration of $20 \mathrm{~m}$-equiv/l. Choline was chosen as the accompanying cation since it is a large ion and so relatively impermeable. Variations in its concentration should not, therefore, produce much diffusion potential. With a change in chloride concentration from 10 to 100 m-equiv/l, the mean potential difference fell (Fig. 2) but the results varied considerably between individuals, from a fall of $10 \mathrm{mV}$ in one individual to a rise of $2 \mathrm{mV}$ in another.

Using various solutions having constant sodium concentration but each with a different anion, the potential difference was measured in five subjects (Table). The solutions were introduced in random order. In Fig. 4 the potential difference observed in one of the individuals has been plotted against the size of the hydrated anion. The halides are similar in ion size and it was found that the potential difference did not vary significantly when different halides were present in the luminal solution. With sulphate and larger anions, the potential differences measured were higher than with the halides but similar to each other. Nitrate and bicarbonate were between these groups both in anion size and in observed potential difference.

OSMOLALITY AND GLUCOSE

In three subjects, the effect of varying the osmolality 


\begin{tabular}{lrrlllllll}
\hline & $\mathrm{Br}^{-}$ & $\mathrm{I}^{-}$ & $\mathrm{NO}_{3}{ }^{-}$ & $\mathrm{HCO}_{3}{ }^{-}$ & Acetate $^{-}$ & $\mathrm{SO}_{4}{ }^{2-}$ & $\mathrm{H}_{3} \mathrm{PO}_{4}{ }^{-}$ & $\mathrm{HPO}_{4}{ }^{2-}$ & $\mathrm{Citrate}^{-}$ \\
\hline Mean change & 1.0 & 0.6 & $-3.5^{2}$ & -2.1 & -5.4 & -7.4 & -6.8 & -9.0 & -5.6 \\
SD & \pm 1.4 & \pm 1.5 & \pm 1.1 & \pm 2.2 & \pm 3.2 & \pm 2.5 & \pm 3.6 & \pm 3.2 & \pm 4.1 \\
\hline
\end{tabular}

Table Change in potential difference on substituting various anions for chloride ${ }^{1}$

${ }^{1}$ Sodium concentration was 150 m-equiv/l (five subjects mean $\pm 1 \mathrm{SD}$ ).

${ }^{2}$ A negative sign indicates that the luminal surface became more negative.

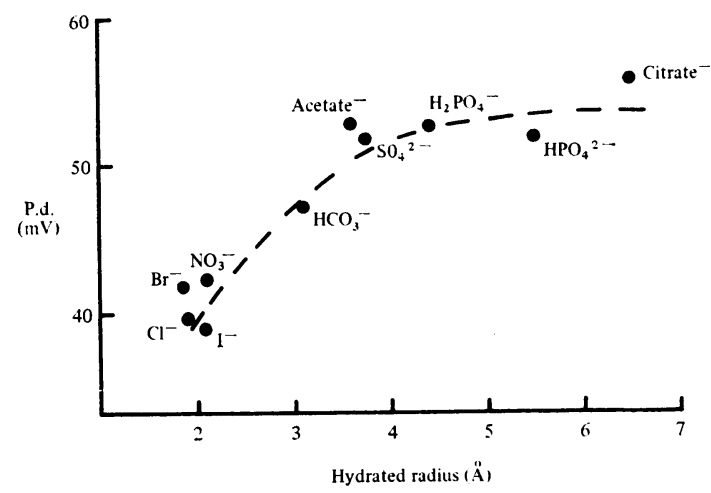

Fig. 4 Effect on the potential difference of altering the anion accompanying sodium. The results are shown for one of the subjects. Mannitol was added when necessary to maintain isotonicity.

of the luminal solution was examined. A solution of sodium sulphate 20 m-equiv/l was used and its content of mannitol varied to provide osmolalities of 100 to $400 \mathrm{~m} \mathrm{Osm}$. Over this range no significant variations in potential difference were observed. Similar experiments were done using mannitol and glucose in the luminal solution but with the solutions isotonic. With glucose concentrations in the lumen up to $80 \mathrm{~m}$ Moles $/ \mathrm{l}$, there was no significant variation of potential difference.

\section{Discussion}

The potential difference of skin probably depends primarily on the activitiy of the sweat glands (Edelberg, 1968), and, as these are almost universally distributed over human skin, a suitable choice of site for the reference electrode may mean a lower skin potential difference but does not eliminate it. Perianal skin for example, has a relatively low potential difference possibly because it is usually a moist area with low electrical resistance. The present results show that the skin potential difference can be consistently eliminated by the simple procedure of injecting saline into the skin. This is probably because there results a considerable flow of ions through the stratum germinativum, thereby pro- ducing a fall in electrical resistance and effectively 'shorting' the skin potential difference (Fig. 5). Thus, direct measurement of the transmucosal potential difference of rectum and colon is possible without recourse to intravenous or subcutaneous electrodes.

The measurements with different solutions showed that the observed potential difference could be considerably influenced by the ionic composition of the solution bathing the luminal side. The experiments with various anions indicated that it was size rather than chemical nature which determined their effects on the potential difference which had its greatest value when the anion radius exceeded 3.5 to $4 \mathrm{~A}^{\circ}$. A likely interpretation is that the luminal anions influence the transmucosal potential difference to the extent to which they penetrate the epithelium and so act as an electrical 'shunt' (Fig. 5). Thus with larger, relatively impermeable anions in the lumen, the 'shunt' is reduced and so the potential difference is higher. The findings are consistent with the hypothesis that anions must pass through a restrictive barrier having pores of about 3 to $4 \mathrm{~A}^{\circ}$ diameter. Other observations (Edmonds and Pilcher, 1971), showing that bulk movement of water induced by osmotic gradients does not affect sodium movement in this part of intestine, also suggest that the ions pass through pores of about this size and so are in agreement with the present results.

The results of changing the concentration of the cation in the lumen showed that sodium was the most important cation influencing the magnitude of the potential difference. A similar dependence of colonic potential difference on sodium concentration has been observed in other species (Gilles-Baillien and Schoffeniels, 1967; Edmonds and Marriott, 1968). It was especially obvious if the potential difference was increased after the subjects had been given mineralocorticoids. The influence of sodium was most apparent when sulphate was used as the accompanying anion as then the shunting effect, which occurs when permeating anions like chloride are in the lumen, is reduced. The mucosa of the human rectum can absorb sodium from the lumen against considerable electrochemical gradients indicating the presence of an active transport system (Edmonds, 1971) and the rest of the colon appears to 


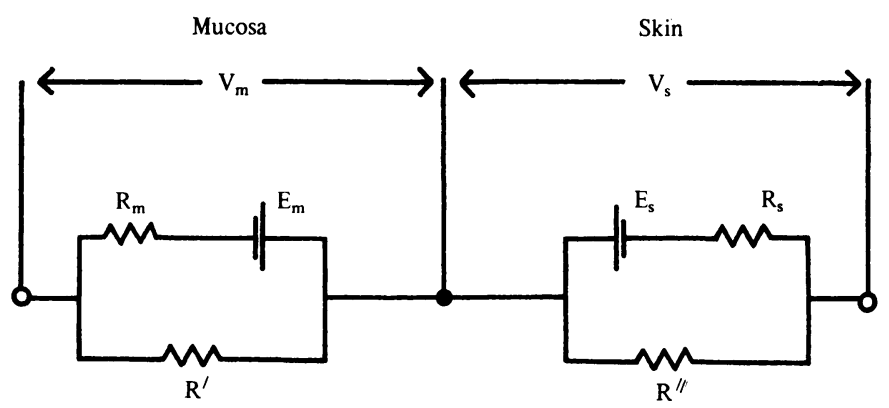

Fig. 5 Equivalent circuit of intestinal mucosa and skin. The simple circuits give a good approximation to the behaviour of the epithelia. (Edelberg, 1968; Edmonds and Marriott, 1968).

The skin potential difference $(V s)$ and the mucosal potential differences $(V m)$ depend both on sources of electromotive force ( $E s$ and Em), which probably result from active ionic transport, and on the shunt resistances $\left(R^{\prime}\right.$ and $\left.R^{\prime \prime}\right)$. Elimination of $V s$ by intradermal injection of saline was probably a result of $R^{\prime \prime}$ being reduced to a very low value. The rise of $\mathrm{Vm}$ observed when a relatively impermeable anion, eg, sulphate, was substituted for chloride was probably due to an increase in $R^{\prime}$.

have similar properties (Levitan, Fordtran, Burrows, and Ingelfinger, 1962; Duthie and Atwell, 1963; Devroede and Phillips, 1969). The evidence of animal experiments suggests that an electrogenic sodium 'pump' is responsible, the transfer of sodium ions electrically polarizing the epithelium (Edmonds and Marriott, 1968) and most of the other permeable ions moving passively along the electrochemical gradients. The amount of sodium being absorbed would therefore be an important factor in determining the magnitude of the potential difference. Our results are consistent with this view. Thus, mineralocorticoids increase the sodium absorption rate (Edmonds and Pilcher, 1971) and, as expected, increase the potential difference. Reducing the luminal sodium concentration, on the other hand, reduces the amount of sodium absorbed and, as observed, causes a fall in potential difference. However, as our results and those of Dalmark (1970) showed, an appreciable potential difference remains even when the luminal sodium concentration is reduced almost to zero. This suggests that at least in the circumstances of low luminal sodium concentration, other mechanisms, possibly bicarbonate secretion, for example, may play a part in producing the electrical polarization.

An alternative possibility to that outlined above is that the fall in potential difference which accompanied reduction of luminal sodium concentration was a diffusion potential. These potentials, a consequence of differing rates of diffusion of ions moving through solutions or membranes, may be present in any system, living or not living, if solutions of different ionic composition or strength are allowed to diffuse into each other. If the effects of changes in luminal sodium concentration were accounted for by a sodium diffusion potential, then this would also seem consistent with the hypothesis that aldosterone acts by increasing the tissue permeability to sodium (Crabbé and de Weer, 1964; Sharp and Leaf, 1966). Since, however, colonic epithelium is permeable to ions other than sodium, the transepithelial potential difference will depend upon relative permeabilities. The probable effects are suggested by consideration of the constant field equation (Goldman, 1943; Hodgkin and Katz, 1949) which gives the diffusion potential, E, expected across a membrane possessing different permeabilities to the various ions in the bathing solutions in relationship to their concentrations:

$$
\mathrm{E}=2 \cdot 3 \frac{\mathrm{RT}}{\mathrm{F}} \log \frac{\mathrm{P}_{\mathrm{K}}[\mathrm{K}]_{\mathrm{m}}+\mathrm{P}_{\mathrm{Na}}[\mathrm{Na}]_{\mathrm{m}}+\mathrm{P}_{\mathrm{Cl}}[\mathrm{Cl}]_{\mathrm{s}}}{\mathrm{P}_{\mathrm{K}}[\mathrm{K}]_{\mathrm{s}}+\mathbf{P}_{\mathrm{Na}}[\mathrm{Na}]_{\mathrm{s}}+\mathrm{P}_{\mathrm{Cl}}[\mathrm{Cl}]_{\mathrm{m}}}
$$

where $\mathbf{P}$ is the relative ionic permeability coefficient, the subscripts $\mathrm{m}$ and $\mathrm{s}$ refer here to the luminal solution and the extracellular fluid respectively, $R$ is the gas constant, $T$ the absolute temperature, and $F$ the faraday. The activity coefficients have been omitted as in most of our experiments the ionic strengths of the luminal and extracellular fluids were similar. Although the equation was derived for a simpler system than a membrane as complex as a layer of cells, it has proved useful in practice in interpreting the effects of potential difference in similar 
situations, for example, with gall bladder (Diamond, 1962). From the equation it is evident that if changes of sodium concentration are to produce marked potential difference effects simply by diffusion gradients, the membrane must be considerably more permeable to sodium (that is, $\mathbf{P N}_{\mathbf{a}}>\mathbf{P}_{\mathrm{K}}$ or $\mathbf{P}_{\mathrm{Cl}}$ ) and the relative permeability to sodium must increase when mineralocorticoids are given. Yet these predictions are not substantiated by observations. First, studies on colonic epithelium in animals and man suggest that the permeabilities to sodium, potassium, and chloride differ little (Curran and Schwartz, 1960; Shields, 1966). Secondly, no significant change in electrical conductivity or increase in sodium conductance is observed when colonic epithelium is exposed to aldosterone (Cofré and Crabbé, 1967; Edmonds and Marriott, 1970). Finally, in some recent investigations, we have found that sodium permeability of rectal epithelium is less than that of potassium or chloride. It is reasonable to conclude therefore that the sodium diffusion potential contributes little to the change in potential difference observed when the luminal sodium concentration is altered. In the case of the effects of changing potassium and chloride concentration, however, these were small and consistent with their arising as diffusion potentials.

Finally, one other process which may contribute to transepithelial potentials must be briefly considered. When an osmotic gradient exists across a tissue, the flow of fluid induced may produce a potential difference (Diamond, 1962; Diamond and Harrison, 1966) and for small intestinal epithelium in vitro such potential differences of appreciable magnitude have been demonstrated (Smyth and Wright, 1966). These osmotically induced potential differences (often called streaming potentials) have been attributed to water flow through pores having fixed charges on their walls. We found, however, no evidence of osmotically induced potentials in the lower sigmoid colon or rectum.

One of us (E.Q.A.) was supported by a grant from the Commonwealth Fellowship Commission administered by the British Council. We wish to record our grateful thanks to Professor C. G. Clarke for his encouragement and advice.

\section{References}

Anderssen, S., and Grossman, M. I. (1965). Profile of pH, pressure and potential difference at the gastroduodenal junction in man. Gastroenterology, 49, 364-371.

Cofré, G., Crabbé, J. (1967). Active sodium transport by the colon of Bufo marinus: stimulation by aldosterone and antidiuretic hormones. J. Physiol. (Lond.), 188, 177-190.

Crabbe, J., and de Weer, P. (1964). Action of aldosterone on the bladder and skin of the toad. Nature (Lond.), 202, 298-299.

Curran, P. F., and Schwartz, G. F. (1960). Na, Cl, and water transport by rat colon. J. gen. Physiol., 43, 555-571.

Dalmark, M. (1970). The transmucosal electrical potential difference across the human rectum in vivo following perfusion of different electrolyte solutions. Scand. J. Gastroent., 5, 421-426.

Devroede, G. J., and Phillips, S. F. (1969). Studies of the perfusion technique for colonic absorption. Gastroenterology, 56, 92-100.

Diamond, J. M. (1962a). The reabsorptive function of the gall bladder. J. Physiol. (Lond.), 161, 442-473.

Diamond, J. M. (1962b). The mechanism of water transport by the gall bladder. J. Physiol. (Lond.), 161, 503-527.

Diamond, J. M., and Harrison, S. C. (1966). The effect of membrane fixed charges on diffusion potentials and streaming potentials. J. Physiol. (Lond.), 183, 37-57.

Donaldson, P. E. K. (1958). Electronic Apparatus for Medical Research, p. 569. Butterworths, London.

Duthie, H. L., and Atwell, J. D. (1963). The absorption of water, sodium and potassium in the large intestine with particular reference to the effects of villous papillomas. Gut, 4, 373-377.

Edelberg, R. (1968). Biopotentials from the skin surface: the hydration effect. Ann. N.Y. Acad. Sci., 148, 252-262.

Edmonds, C. J. (1970). Electrical potentials of the sigmoid colon and rectum in irritable bowel syndrome and ulcerative colitis. Gut, 11, 867-874.

Edmonds, C. J. (1971). Absorption of sodium and water by human rectum measured by a dialysis method. Gut, 12, 356-362.

Edmonds, C. J., and Godfrey, R. (1970). Measurement of electrical potentials of the human rectum and pelvic colon in normal and aldosterone-treated patients. Gut, 11, 330-337.

Edmonds, C. J., and Marriott, J. (1968). Electrical potential and short circuit current of an in vitro preparation of rat colon mucosa. J. Physiol. (Lond.), 194, 479-494.

Edmonds, C. J., and Marriott, J. (1970). Sodium transport and short circuit current in rat colon in vivo and the effect of aldosterone. J. Physiol. (Lond.), 210, 1021-1039.

Edmonds, C. J., and Pilcher, D. (1971). Sodium transport mechanisms of the large intestine. In Transport Across the Intestine (A Glaxo Symposium), pp. 43-59, edited by W. L. Burland and P. D. Samuel. Churchill, London.

Geall, M. G., Spencer, R. J., and Phillips, S. F. (1969). Transmural electrical potential difference of human colon. Gut, 10, 921-923.

Gilles-Baillien, M., and Schoffeniels, E. (1967). Bioelectric potentials in the intestinal epithelium of the Greek tortoise. Comp. Biochem. Physiol., 23, 95-104.

Goldman, D. E. (1943). Potential, impedance and rectification in membranes. J. gen. Physiol., 27, 37-60.

Grantham, R. N., Code, C. F., and Schlegel, J. F. (1970). Reference electrode sites in determination of potential difference across the gastroesophageal mucosal junction. Mayo Clin. Proc., 45, 265-274.

Hodgkin, A. L., and Katz, B. (1949). The effect of sodium ions on the electrical activity of the giant axon of the squid. J. Physiol. (Lond.), 108, 37-77.

Levitan, R., Fordtran, J. S., Burrows, B. A., and Ingelfinger, F. J. (1962). Water and salt absorption in the human colon. J. clin. Invest., 41, 1754-1759.

Sharp, G. W. G., and Leaf, A. (1966). Mechanism of action of aldosterone. Physiol. Rev., 46, 593-633.

Shields, R. (1966). Absorption and secretion of electrolytes and water by the human colon with particular reference to benign adenoma and papilloma. Brit. J. Surg., 53, 893-897.

Smyth, D. H., and Wright, E. M. (1966). Streaming potentials in the rat small intestine. J. Physiol. (Lond.), 182, 591-602. 